\section{September 2001}

European Association for

Osseointegration Congress 2001

Date: $13.09 .01-16.10 .01$

Venue: Milan.

Contact: Annie Nagem, EAO Office,

1 Wimpole Street, London.

W1M 8AE.

Tel: +44 (0)20 72903948

5th BAAD (British Academy of Aesthetic

Dentistry) Open Meeting and 7th

Scientific Meeting

Date: 20.09.01 - 22.09.01

Venue: Celtic Manor Hotel, Cardiff

Contact: The Conference Shop,

94 Felpham Road, Felpham, West Sussex.

PO22 7PG.

Tel: $+44(0) 1243841999$

Fax: +44 (0) 1243841842

E-mail: sales@conferenceshop.com

FDI World Dental Congress 2001

Date: 27.09.01-01.10.01

Venue: Kuala Lumpur

Contact: FDI, 7 Carlisle Street,

London W1V 5RG

Tel: +44 (0)20 79357852

Fax: +44 (0)20 74860183

Website: www.fdi.org.uk

\section{British Orthodontic Conference}

Date: 30.09.01 - 03.10.01

Venue: Harrogate International Centre

Contact: Mrs Ann Wright, British

Orthodontic Conference, BOS Office, 291

Gray's Inn Road, London WC1X 8QF.

Tel: +44 (0)20 78372193

Fax: +44 (0)20 78372193

E-mail: awrightbos@msn.com

\section{October 2001}

Australasian Osseointegration Society

Biennial Conference

Date: 11.10.01-14.10.01

Venue: The Sheraton Perth Hotel, Perth,

Australia

Contact: EventEdge International Man- agement Group, PO Box 749, Wembley,

Australia, WA 6913

Tel: ++61 0894894800

Fax: ++61 0894894801

E-mail:info1@eventedge.com.au/osseo

52nd Annual Session of the American

Academy of Oral and Maxillofacial

Radiology

Date: 3.10.01-07.10.01

Venue: Benson Hotel, Portland, USA.

Contact: Dr. M. Kevin O Carroll, Executive

Director, The American Academy of Oral

and Maxillofacial Radiology, PO Box

55722, Jackson, MS 39296.

Tel: (601) 9846060

Fax: (601) 9846086

E-mail: mocarroll@sod.umsmed.edu

ADI International Implant Congress:

Building Foundations for Aesthetic

Implant Solutions

Date: $12.10 .01-13.10 .01$

Venue: London Heathrow Marriott Hotel.

Contact: Mrs Cherry Wilson, ADI Secre-

tariat, 37 Halford Road, Richmond, Surrey.

Tel: +44 (0)2083320321

Fax: +44 (0)2089400337

E-mail: adi.uk@btinternet.com

SAAD Annual Conference Day

Date: 13.10 .01

Venue: King's Fund, London.

Contact: The Conference Secretary, SAAD,

53 Wimpole Street, London, W1G 8YH.

Tel: +44 (0)2079351656

Web-site: www.saaduk.org

Oral Health Promotion Research Group

Tenth Scientific Meeting

Date: 31.10 .01

Venue: University of London.

Contact: Stella Kwan, Lecturer in Dental

Public Health, Leeds Dental Institute,

Clarendon Way, Leeds LS2 9LU.

Tel: $+44(0) 1132336329$

Fax: +44 (0)1132336140

E-mail: S.Kwan@leeds.ac.uk

\section{November 2001}

University of Sheffield Restorative Dentistry 19th Annual Postgraduate Day Managing Challenging Restorative

Patients

Date: 10.11 .01

Venue: Sheffield Hallam University

Convention Centre

Contact: Peter Johnson, event organiser, School of Clinical Dentistry, Claremont Crescent, Sheffield S10 2TA.

Tel: +44 (0)114266 0987

Fax: +44 (0)114266 5326

Faculty of General Dental Practitioners (UK): Who manages your practice? Date: 02.11 .01

Venue: The Royal College of Surgeons of England, London

Contact: Catherine Elkington, FGDP

(UK), 35-43 Lincoln's Inn Fields, London, WC2A 3PE.

Tel: +44 (0) 12078696758

Fax: +44 (0)12078696765

Faculty of General Dental Practitioners (UK) \& GKT Dental Institute: Developing a Restorative Research Project

Date: 07.11 .01

Venue: GKT Denmark Hill, London Contact: Catherine Elkington, FGDP (UK), 35-43 Lincoln's Inn Fields, London, WC2A 3PE.

Tel: +44 (0)2078696758

Fax: +44 (0)12078696765

\section{January 2002}

The Arab Health Series Exhibition and Conference

Date: 26.01.02-29.01.02

Venue: Dubai World Trade Centre.

Contact: Elaine Nettleton, PR Manager,

IIR Exhibitions, PO Box 28943, Dubai.

Tel: 97143365161

Fax: 97143360137

E-mail: enettleton@iir-dubai 